Arq. Bras. Med. Vet. Zootec., v.66, n.5, p.1359-1366, 2014

\title{
Migração espermática em suínos após inseminação artificial intrauterina profunda
}

\author{
[Sperm migration in pigs after deep intrauterine artificial insemination] \\ F.R.C.L. Almeida ${ }^{1}$, V.A. Gheller ${ }^{2}$, P.A. Auler ${ }^{1}$, G.H.F.A. Moreira ${ }^{2}$, \\ R.B.C. Jardim ${ }^{1}$, J.M. Silva Filho ${ }^{2}$ \\ ${ }^{1}$ Instituto de Ciências Biológicas -UFMG - Belo Horizonte, MG \\ ${ }^{2}$ Escola de Veterinária - UFMG - Belo Horizonte, MG
}

\begin{abstract}
RESUMO
A inseminação artificial intrauterina profunda (IIP) é de grande importância para a indústria suinícola, em função do maior número de doses produzidas por reprodutores de alto mérito genético e da possibilidade da utilização de biotecnologias, como sêmen sexado e/ou congelado. Entretanto, necessita-se compreender com maior propriedade os mecanismos pelos quais os espermatozoides colonizam as tubas uterinas. Assim sendo, pretende-se com o presente experimento avaliar a existência ou não de migração intraperitoneal de espermatozoides inseminados profundamente em um dos cornos uterinos, mediante a obtenção de oócitos fertilizados no corno contralateral à inseminação e seccionado na base, na junção com o corpo do útero. Quatorze fêmeas pluríparas foram divididas em dois grupos experimentais, sendo que em um deles as fêmeas foram submetidas à secção da base de um dos cornos uterinos (Grupo Operado, $n=7)$, enquanto as do Grupo Controle $(n=7)$ não foram submetidas a nenhuma intervenção cirúrgica. Ambos os grupos foram submetidos à IIP, sendo as fêmeas abatidas $5 \pm 1,2$ dias após a última inseminação. Os sistemas genitais das fêmeas foram coletados, dissecados e o número de corpos lúteos contados em ambos os ovários. A recuperação dos embriões foi feita por meio de lavagem das tubas e cornos uterinos com solução de PBS (Phosphate Buffered Saline), após o que se avaliou os fluidos coletados em lupa para a identificação de embriões. Em ambos os grupos experimentais, foram encontrados embriões nos segmentos do sistema genital de ambos os lados. Apenas uma fêmea apresentou embriões nos segmentos em somente um dos lados no grupo operado. Diante dos resultados aqui observados, concluiu-se que a migração espermática no suíno pode ocorrer tanto por via retrógrada pelo útero quanto por migração intraperitoneal. Estes achados certamente contribuirão para aumentar a eficiência da técnica de IIP, sendo de grande valia para o aprimoramento da indústria suinícola.
\end{abstract}

Palavras-chave: suíno, inseminação intrauterina profunda, migração espermática

\begin{abstract}
Deep intrauterine insemination (DUI) is of great importance for the swine industry as it can increase the efficiency in the use of boars of high genetic merit, and facilitate the use of biotechnologies such as frozen and sexed semen. However, a better understanding of the mechanisms by which the sperm colonize the uterine tubes is essential. The aim of the present study was to investigate the existence of intrauterine sperm migration after DUI in one uterine horn, through the fertilization of oocytes in the contra lateral uterine horn. Fourteen multiparous sows were divided into two experimental groups: Operated $(n=7)$, where females had a segment close to the base of the uterine horn surgically removed, and Control $(n=$ 7), females with intact uterus. Both groups were inseminated through DUI and slaughtered $5 \pm 1.2$ days after the last insemination. The reproductive tracts collected were dissected and the number of corpora lutea counted in both ovaries. Embryo recovery was performed though flushings of uterine tubes and horns with Phosphate Buffered Saline solution and further examination under a dissecting microscope. Embryos were found in the uterine horns of both sides of the reproductive tract in both experimental
\end{abstract}

Recebido em 24 de agosto de 2013

Aceito em 21 de agosto de 2014

E-mail: falmeida@icb.ufmg.br 
groups. In the operated group, just one female had embryos in only one side of the reproductive tract. The results presented herein suggest that sperm migration in pigs may occur both in a retrograde way through the uterus and by intraperitoneal migration. These findings will certainly contribute to increase the efficiency of the DUP technique, which is of great importance for the improvement of the swine industry.

Key-words: pigs, deep intrauterine insemination, sperm migration

\section{INTRODUÇÃO}

A utilização da inseminação artificial (IA) em suínos tem aumentado dramaticamente nos últimos anos (Vazquez et al., 2005), em substituição à monta natural, por ser um procedimento barato, fácil e rápido. Além disso, é um procedimento frequentemente realizado pelos próprios produtores, com obtenção de grande sucesso nos últimos anos (Vazquez et al., 2008). Tradicionalmente, a IA utilizada na espécie suína envolve a utilização de uma dose inseminante de cerca de $3 \times 10^{9}$ espermatozoides, diluídos em um volume que varia de $80-100 \mathrm{~mL}$. A deposição do sêmen é feita na porção posterior do canal cervical (aproximadamente $15 \mathrm{~cm}$ de profundidade) e o número de inseminações varia de duas a três por estro (Vazquez et al., 2008). As múltiplas inseminações estão associadas a um momento da ovulação muito variável em relação ao início do estro, dificultando precisar o momento ideal para a realização da IA, o mais próximo possível do momento da ovulação (Bracken et al., 2003).

Considerando que bilhões de espermatozoides são depositados no canal cervical durante a monta natural ou IA, apenas uma pequena proporção atinge o reservatório espermático (Mburu et al., 1996). Dois mecanismos têm sido responsabilizados pela grande perda de células espermáticas ao longo do trajeto no sistema genital feminino: refluxo de sêmen e fagocitose por leucócitos polimorfonucleares (Vazquez et al., 2005). Sob essas circunstâncias, a utilização de doses inseminantes com baixas concentrações espermáticas poderia estar associada a um aumento nas taxas de gestação se as inseminações fossem realizadas o mais profundamente possível (Vazquez et al., 2005).

Um método de inseminação intrauterina profunda (IIP) foi descrito em suínos, possibilitando a utilização de novas biotecnologias, tais como sêmen criopreservado ou sexado, bem como uma melhor eficiência de doses inseminantes tradicionais, com baixo número de espermatozoides (Martinez et al., 2001a,b; 2002). Esta tecnologia visa depositar o sêmen próximo ao local de fertilização, utilizando um volume muito pequeno. Assim, tal estratégia permite reduzir $\mathrm{o}$ trânsito dos espermatozoides ao longo do útero $\mathrm{e}$, consequentemente, as perdas espermáticas por fagocitose e por refluxo (Vazquez et al., 2005).

Há evidências de que os espermatozoides depositados em um corno uterino possuem capacidade de atingir a tuba uterina contralateral e fertilizar uma alta proporção de oócitos (Martinez et al., 2005; Tummaruk et al., 2007). No entanto, o caminho usado pelos espermatozoides durante essa migração continua incerto. Tummaruk e colaboradores (2007) relataram que os espermatozoides podem ser liberados do reservatório espermático de um lado do sistema genital e atingirem o outro lado por migração transperitoneal, já descrita em bovinos (Larsson, 1986). De acordo com o mesmo autor, há ainda a possibilidade de os espermatozoides serem transportados de um corno uterino ao outro por migração uterina retrógrada. Entretanto, no mesmo trabalho, foi demonstrado que os espermatozoides foram encontrados em apenas um dos lados do sistema genital das fêmeas após IIP, embora os embriões tenham sido encontrados em ambos os lados.

Diante da grande importância da IIP para a suinocultura, maiores investigações são necessárias visando-se entender os mecanismos pelos quais os espermatozoides colonizam as tubas uterinas após a IIP com baixo número de espermatozoides. Portanto, o presente trabalho foi delineado para testar a hipótese de que os espermatozoides depositados profundamente em um dos cornos uterinos possuem capacidade de fertilizar oócitos no corno contralateral através de dois sistemas a saber: tanto por meio de um transporte retrógrado pelo útero quanto por migração transperitoneal. 


\section{MATERIAIS E MÉTODOS}

Quatorze fêmeas pluríparas (DanBred DB90), de $3^{\mathrm{a}}$ a $6^{\mathrm{a}}$ ordens de parto, foram distribuídas aleatoriamente em dois grupos experimentais: 1Controle $(\mathrm{n}=7)$, fêmeas com útero íntegro; 2Operado $(\mathrm{n}=7)$, fêmeas submetidas à secção cirúrgica da base de um dos cornos uterinos.

Com o objetivo de impedir que os espermatozoides depositados profundamente em um dos cornos uterinos, realizassem o caminho retrógrado pelo útero e atingissem a extremidade do corno contralateral ao inseminado, fertilizando oócitos presentes na tuba uterina ipsilateral, realizou-se a secção e posterior ligadura na base de um dos cornos uterinos, por procedimento cirúrgico de laparotomia.

Para o procedimento cirúrgico, as fêmeas permaneceram em jejum alimentar por 24 horas e hídrico por 12 horas. Utilizou-se nas fêmeas anestesia dissociativa com tiletamina e zolazepam (Zoletil® 50, Virbac, Brasil) na dose intramuscular de $0,40 \mathrm{mg} / \mathrm{kg}$. Após a correta assepsia, procedeu-se à exposição uterina por meio de laparotomia no flanco, com incisão na fossa paralombar. A seguir, realizaram-se duas ligaduras do corno uterino direito, na junção com o corpo do útero, a uma distância de $3 \mathrm{~cm}$ uma da outra, utilizando-se fio catgut cromado 1 , padrão Utrech modificado com oclusão total do lúmen do corno. O corno uterino direito foi então seccionado entre as duas ligaduras (Fig. 1 A, B), quando se retirou um segmento, na base do corno uterino, de aproximadamente $1 \mathrm{~cm}$.

Para o tratamento pós-operatório, utilizou-se como antibiótico a Penicilina G Benzatina 40.000UI/kg (Penfort@, Ouro Fino Saúde Animal Ltda, Brasil) por via intramuscular em três aplicações com intervalos de 48 horas. Além disso, utilizou-se como anti-inflamatório flunixin meglumine 2,2mg/kg (Flumedin ${ }^{\circledR}$, Jofadel Indústria Farmacêutica S/A, Brasil) por via intramuscular em três aplicações com intervalo de 24 horas. As fêmeas submetidas ao procedimento cirúrgico foram utilizadas para as inseminações somente após o completo restabelecimento das cirurgias. Os procedimentos de manuseio estão de acordo com os princípios éticos de experimentação animal, adotados e aprovados pelo Comitê de Ética em Experimentação Animal CEUA/UFMG.

As porcas foram inseminadas com duas doses inseminantes de sêmen diluído, resfriado e estocado por um período máximo de 36 horas. A concentração da dose inseminante foi $1 \times 10^{9}$ espermatozoides totais, diluídos em um volume de $20 \mathrm{~mL}$. O procedimento de IIP foi realizado nas fêmeas sem necessidade de sedação, como descrito por Martinez et al. (2002).

Dessa forma, após limpeza da área perineal das fêmeas, uma pipeta comercial (Deep Golden Pig, IMV-Brasil) foi introduzida através da vagina até a cérvix. Posteriormente, um cateter flexível (1,8m de comprimento, diâmetro externo de $4 \mathrm{~mm}$ e interno de $1,8 \mathrm{~mm}$ ) foi introduzido através do orifício da pipeta, movido em direção à cérvix e guiado a um dos cornos uterinos. Em seguida, uma seringa contendo a dose inseminante foi conectada ao cateter flexível, sendo o sêmen depositado vagarosamente na extremidade do corno uterino. Visando eliminar o sêmen residual no cateter de inseminação antes de sua remoção, foram utilizados $5 \mathrm{~mL}$ de diluidor puro para expulsar todo o sêmen restante do interior do mesmo. A inseminação foi considerada correta quando cerca de $150 \mathrm{~cm}$ do cateter foi inserido através da pipeta e sua forma reta mantida após a sua remoção (Martinez et al., 2006).

As fêmeas foram abatidas $5 \pm 1,2$ dias após a última IIP, sendo os sistemas genitais das fêmeas coletados e submetidos ao seguinte protocolo:

a) As tubas uterinas foram ligadas em suas extremidades uterina e infundibular, utilizando fio de algodão (Brasuture®);

b) Os cornos uterinos foram divididos, visualmente, em três porções (cranial, média e caudal; Fig. 2) que foram ligadas com o mesmo fio descrito anteriormente;

c) Finalmente, os sistemas genitais foram identificados, colocados em caixas isotérmicas contendo gelo e transportadas ao laboratório. 


\section{Almeida et al.}

A

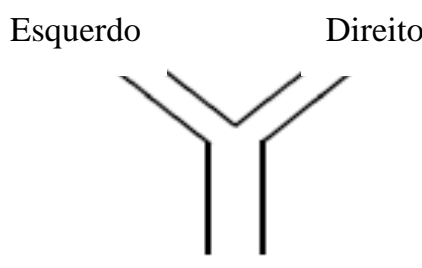

Controle
Esquerdo

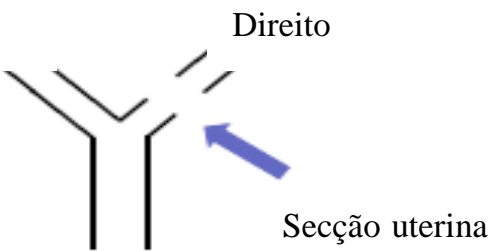

Operado

B

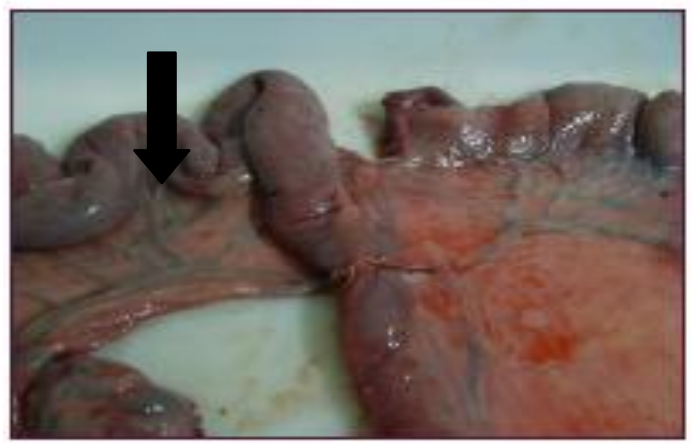

Figura 1A. Desenho esquemático representando o procedimento cirúrgico realizado para secção de um segmento da base de um dos cornos uterinos. B. Detalhe do útero de uma fêmea suína submetida à transecção de um dos cornos uterinos.

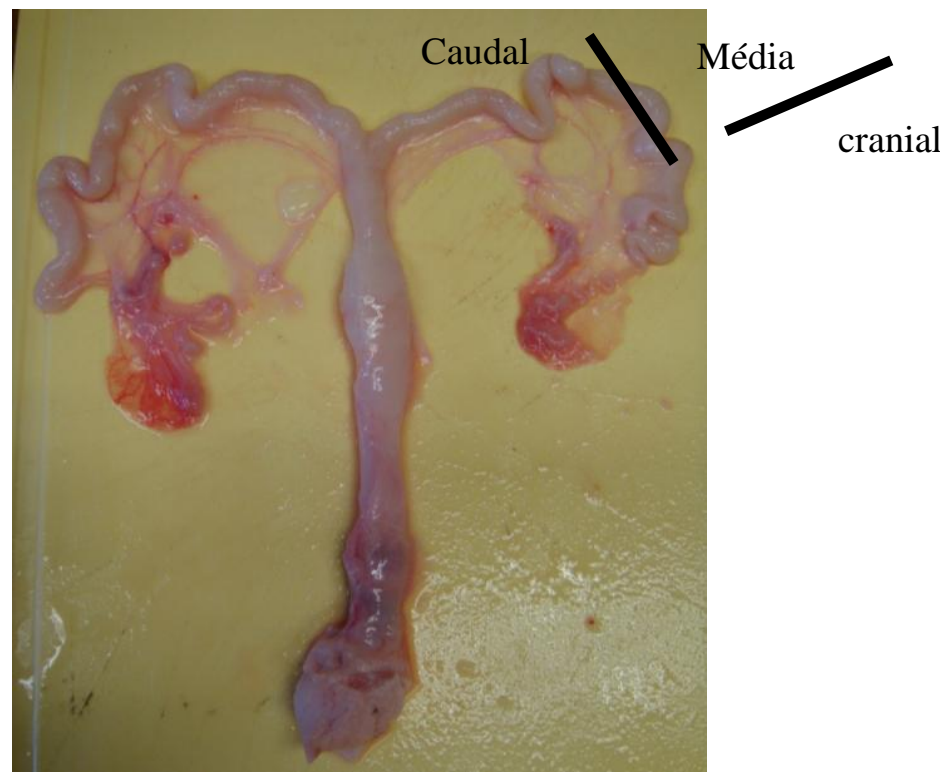

Figura 2. Sistema genital de fêmea suína representando as três porções (cranial, média e caudal) em que foram divididos os cornos uterinos e posteriormente ligados com fio de algodão (Brasuture®). 
No laboratório, os corpos lúteos presentes em ambos os ovários foram contados. Posteriormente, as tubas e cornos uterinos de ambos os lados foram lavados, duas vezes, por meio de injeções de $20 \mathrm{~mL}$ de solução de PBS (Phosphate Buffer Saline) de cada vez, sendo o fluido coletado em placas de Petri previamente identificadas (segmento do sistema genital e lado), perfazendo 16 amostras: quatro oriundas de ambas as tubas uterinas, e 12 dos dois cornos uterinos, divididos em três partes cada um.

Após a lavagem, as placas de Petri foram submetidas a um período de repouso de 15 a 20 minutos, antes de serem examinadas em microscópio estereoscópio. Uma vez identificados os embriões, foram contados e classificados quanto ao grau de desenvolvimento e qualidade, avaliou-se também a presença de oócitos não fertilizados em microscópio de contraste de fase, conforme descrito por Martinez et al. (2006). Uma vez finalizada a contagem, procedeu-se ao cálculo de: a) taxa de recuperação (número total de embriões e oócitos recuperados, em relação ao total de corpos lúteos contados em ambos os ovários X 100); b) presença de fertilização unilateral (embriões recuperados em apenas um corno uterino); c) presença de fertilização bilateral parcial (quando menos de $60 \%$ das estruturas encontradas naquele corno forem embriões); d) taxa de gestação (percentagem de fêmeas com pelo menos quatro embriões viáveis (Martinez et al., 2006).

O delineamento experimental utilizado foi $\mathrm{o}$ delineamento inteiramente casualizado, com dois tratamentos e com um animal por unidade experimental. As variáveis quantitativas analisadas tais como número de corpos lúteos, número total de embriões, taxa de recuperação e taxa de gestação foram analisadas para normalidade e homocedasticidade do resíduo, sendo posteriormente submetidas à análise de variância (ANOVA) e as médias comparadas pelo teste F. Os dados qualitativos (presença de fertilização uni- ou bilateral) foram analisados por distribuição de frequência e aplicado o teste Qui-quadrado para a detecção de eventuais diferenças ente as variáveis avaliadas.

Todas as análises estatísticas foram processadas pelo programa estatístico Statistical Analysis System (SAS), sendo o nível de significância adotado de $95 \%(\mathrm{P}<0,05)$.

\section{RESULTADOS}

Em 5/14 (35,7\%) das 14 fêmeas inseminadas não foram encontrados embriões nos respectivos fluidos das tubas e cornos uterinos, sendo três do grupo Controle e duas do grupo Operado. Assim, a taxa de gestação calculada foi de $57,1 \%$ e $71,4 \%$, para os grupos Controle e Operado, respectivamente (Tab. 1), não havendo, portanto, diferenças estatisticamente significativas entre os tratamentos.

A avaliação dos fluidos ao microscópio estereoscópio revelou a presença de embriões nos segmentos de ambos os lados do sistema genital, bem como nos dois grupos experimentais ( $\mathrm{n}=4$, grupo Controle; $\mathrm{n}=4$, grupo Operado). Os embriões encontrados apresentaram-se em diferentes estágios de desenvolvimento, desde duas/quatro células até mórula (Fig. 2). Apenas uma fêmea do grupo Operado apresentou embriões somente no segmento contralateral ao corno uterino operado (Tab. 1). O número de embriões recuperados nas fêmeas de ambos os grupos experimentais está apresentado na Tab. 2.

Tabela 1. Taxa de gestação e ocorrência de fertilização uni- ou bilateral em fêmeas suínas após inseminação intrauterina profunda

\begin{tabular}{ccccc}
\hline Grupos & $\mathrm{n}$ & Taxa de gestação & Fertilização Bilateral & $\begin{array}{c}\text { Fertilização } \\
\text { Unilateral }\end{array}$ \\
\hline & & $4 / 7$ & $4 / 4$ & $0 / 0$ \\
Controle $^{*}$ & 7 & $(57,1)$ & $(100)$ & $(0)$ \\
& & $5 / 7$ & $4 / 5$ & $1 / 5$ \\
Operado $^{*}$ & 7 & $(71,4)$ & $(80)$ & $(20)$ \\
\hline
\end{tabular}

*Valores apresentados como [n (\%)] 
Tabela 2. Número de embriões suínos recuperados nos cornos uterinos direito e esquerdo das fêmeas de ambos os grupos experimentais

\begin{tabular}{lccc}
\hline Fêmea & Tratamento & Corno direito & Corno esquerdo \\
\hline 1 & Controle & 2 & 2 \\
2 & Controle & 6 & 4 \\
3 & Controle & 0 & 0 \\
4 & Controle & 8 & 4 \\
5 & Controle & 12 & 7 \\
6 & Operado & 0 & 4 \\
7 & Operado & 4 & 11 \\
8 & Operado & 4 & 12 \\
9 & Operado & 1 & 4 \\
10 & Operado & 2 & 6 \\
\hline
\end{tabular}
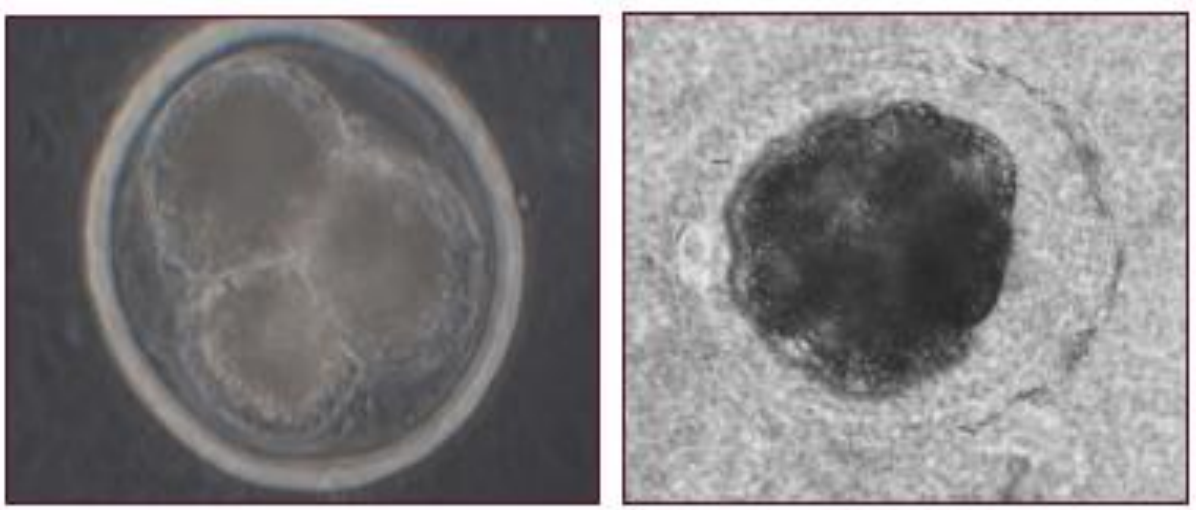

Figura 2 Imagens de embriões coletados dos cornos uterinos de porcas submetidas à inseminação intrauterina profunda

O número de corpos lúteos em ambos os ovários e o número total de embriões recuperados nas tubas e cornos uterinos nos lados direito e esquerdo do sistema genital estão representados na Tab. 3. Após o cálculo da taxa de recuperação, os dados revelaram uma variação de 30 a $50 \%$ nos diferentes tratamentos e lados do sistema genital, não havendo diferenças $(\mathrm{P}>0,05)$ entre ambos os grupos experimentais (Tab. 3).

Tabela 3 Dados obtidos à dissecação e lavagem dos segmentos do sistema genital suíno em ambos os grupos experimentais (médiaterro padrão)

\begin{tabular}{lrrrr} 
& \multicolumn{2}{c}{ Grupo Controle $(\mathrm{n}=4)$} & \multicolumn{2}{c}{ Grupo Operado $(\mathrm{n}=5)$} \\
\cline { 2 - 5 } Parâmetros & \multicolumn{1}{c}{ Direito } & Esquerdo & \multicolumn{1}{c}{ Direito } & \multicolumn{1}{c}{ Esquerdo } \\
\hline No. corpos lúteos & $11,6 \pm 1,6$ & $10,8 \pm 2,0$ & $10,2 \pm 1,6$ & $12,8 \pm 2,0$ \\
No. embriões recuperados & $5,6 \pm 2,0$ & $3,4 \pm 1,4$ & $3,6 \pm 2,0$ & $6,0 \pm 1,4$ \\
Taxa recuperação $(\%)^{*}$ & $50,7 \pm 17,0$ & $36,3 \pm 13,0$ & $28,7 \pm 17,0$ & $49,2 \pm 13,0$ \\
\hline
\end{tabular}

"Taxa de recuperação = número total de embriões e oócitos coletados/número corpos lúteos X 100

\section{DISCUSSÃO}

Os resultados apresentados no presente trabalho confirmam a hipótese de que os espermatozoides depositados profundamente em um dos cornos uterinos possuem a capacidade de fertilizar oócitos no corno contralateral por meio de um transporte tanto retrógrado pelo útero quanto por migração transperitoneal. A migração transperitoneal na espécie suína também foi observada por Hunter (1978), que relatou transporte espermático e fertilização de oócitos 
após a deposição intraperitoneal do sêmen suíno. Além disso, demonstrou-se anteriormente que os espermatozoides poderiam passar através da tuba uterina para a cavidade abdominal durante as primeiras horas após uma inseminação intracervical (Viring e Einarsson, 1981). A existência da migração espermática transperitoneal foi também comprovada em outras espécies domésticas tais como bovinos (Larsson, 1986; Lopez-Gatius, 1995), caprinos (Gonzales, 1972) e ovinos (Negobatikov et al., 1981).

No presente estudo, o procedimento cirúrgico de secção e posterior ligadura na base de um dos cornos uterinos, realizado por meio de laparotomia, atuou como um obstáculo aos espermatozoides depositados profundamente em um dos cornos uterinos no que se refere ao seu caminho retrógrado pelo útero para atingir a extremidade do corno uterino contralateral ao inseminado. Portanto, a presença de embriões de pelo menos duas-células no corno operado comprova a existência de migração transperitoneal. Por outro lado, no grupo Controle, a presença de embriões em ambos os cornos uterinos demonstra claramente a migração espermática por via retrógrada pelo útero e confirma a presença de fertilização bilateral. Tais resultados encontram respaldo no estudo de Tummaruk et al. (2007), que também encontraram embriões em ambas as tubas uterinas e porções proximais dos cornos uterinos, 48 a 72 horas após a inseminação. Em um estudo recente, observou-se migração espermática transuterina mas não transperitoneal em suínos após inseminação intrauterina profunda quando da utilização de uma dose inseminante com baixa concentração espermática $\left(60 \times 10^{6}\right.$ espermatozoides). Segundo os autores, embora esta baixa concentração tenha sido eficiente para IIP, os espermatozoides foram incapazes de migrar para a tuba uterina contralateral pela cavidade peritoneal (Brüssow et al., 2011). Assim sendo, excetuando o estudo de Hunter (1978), o presente trabalho é o mais recente a demonstrar com sucesso a fertilização de oócitos por migração espermática transperitoneal na espécie suína.

\section{CONCLUSÃO}

o presente estudo fornece evidência experimental de que a migração espermática na espécie suína após inseminação intrauterina profunda se dá tanto por via retrógrada pelo útero quanto por via peritoneal. Estes achados contribuirão para aumentar a eficiência da técnica de IIP, sendo de grande valia para o aprimoramento da indústria suinícola.

\section{AGRADECIMENTOS}

Suporte financeiro para o desenvolvimento do presente projeto foi concedido pela FAPEMIG (CVZ-APQ-01712-09). Aos funcionários da Fazenda Experimental Prof. Hélio Barbosa, Nenego e Pedro, por não medirem esforços para o bom andamento do experimento.

\section{REFERÊNCIAS}

BRACKEN, C.J.; SAFRANSKI, T.J.; CANTLEY, T.C. et al. Effect of time of ovulation and sperm concentration on fertilization rate in gilts. Theriogenology, v.60, p.669-676, 2003.

BRÜSSOW, K.P.; TORNER, H.; RÁTKY, J. Sperm migration in pigs after deep intrauterine and intraperitoneal insemination. J. Reprod. Dev., v.57, p.342-345, 2011.

GONZALES, C.S. Intraperitoneal insemination in goats. In: INTERNATIONAL CONGRESS IN ANIMAL REPRODUCTION AND AI, 7, 1972, Munique. Anais... Munique: 1972, v.7, p.1501-1506 (Resumo).

HUNTER, R.H.F. Intraperitoneal insemination, sperm transport and capacitation in the pig. Anim. Reprod. Sci., v.1, p.167-179, 1978.

LARSSON, B. Transperitoneal migration of spermatozoa in heifers. J. Vet. Med. A, v.33, p.714-718, 1986.

LOPEZ-GATIUS, F. Intraperitoneal insemination in repeat-breeder cows: a preliminary report. Theriogenology, v.44, p.153-158, 1995.

MARTINEZ, E.A.; VAZQUEZ, J.M.; ROCA, J. et al. Successful non-surgical deep intrauterine insemination with small numbers of spermatozoa in sows. Reproduction, v.122, p.289-296, 2001a. 
MARTINEZ, E.A.; VAZQUEZ, J.M.; ROCA, J. et al. Deep intrauterine insemination and embryo transfer in pigs. Reproduction, v.58, p.301-311, $2001 b$.

MARTINEZ, E.A.; VAZQUEZ, J.M.; ROCA, J. et al. Minimum number of spermatozoa required for normal fertility after deep intrauterine insemination in non-sedated sows. Reproduction, v.123, p.163-170, 2002.

MARTINEZ, E.A.; VAZQUEZ, J.M.; ROCA, J. et al. An update on reproductive technologies with potential short-term application in pig production. Reprod. Dom. Anim., v.40, p.300309,2005

MARTINEZ, E.A.; VAZQUEZ, J.M.; PARRILLA, I. et al. Incidence of unilateral fertilization after low dose deep intrauterine insemination in spontaneously ovulation sows under Field conditions. Reprod. Dom. Anim., v.41, p.41-47, 2006.

MBURU, J.N.; EINARSSON, S.; LUNDEHEIM, N. et al. Distribution, number and membrane integrity of spermatozoa in the pig oviduct in relation to spontaneous ovulation. Anim. Reproduct. Sci., v.45, p.109-121, 1996.
NEGOBATIKOV, G.; ZIRNOKLEEV, V., ZARUDNEV, S. An experiment on paragenital insemination of sheep. Zhivotnovodstvo, v.1, p.54-56, 1981.

TUMMARUK, P.; SUMRANSAP, P.; TECHAKUMPHU, M. et al. Distribution of spermatozoa and embryos in the female reproductive tract after unilateral deep intra uterine insemination in the pig. Reprod. Dom. Anim., v.42, p.603-609, 2007.

VAZQUEZ, J.M.; MARTINEZ, E.A.; ROCA, J. et al. Improving the efficiency of sperm Technologies in pigs: the value of deep intrauterine insemination. Theriogenology, v.63, p.536-547, 2005.

VAZQUEZ, J.M.; ROCA, J.; GIL, M.A. et al. New developments in low-dose insemination technology. Theriogenology, v.70, p.1216-1224, 2008.

VIRING, S.; EINARSSON, S. Sperm distribution within the genital tract of naturally inseminated gilts. Nord. Vet. Med., v.33, p.145-149, 1981. 